Conclusions: Biomarkers are able to improve prediction of radiographic spinal progression in axSpA, especially if used in addition to the clinical parameters, but the added value seems to be rather small.

Disclosure of Interest: None declared

DOI: 10.1136/annrheumdis-2018-eular.3795

\section{FRI0155 GENES REGULATING BONE HOMEOSTASIS ARE DIFFERENTIALLY EXPRESSED AT PERIPHERAL VERSUS AXIAL ENTHESIAL SITES IN AN ANIMAL MODEL OF SPONDYLOARTHRITIS}

\section{Z. Stavre, Y. Maeda, T. Huang, E. Gravallese. Department of Medicine- Rheumatology, University of Massachusetts Medical School, Worcester, USA}

Background: In spondyloarthritis (SpA), mechanisms of enthesial bone formation are not well understood. Currently, no treatment can reverse this process, although TNF inhibitors may modify bone formation if initiated early. SKG mice exhibit features of $\mathrm{SpA}$ with both peripheral joint and axial inflammation after induction by curdlan via the Dectin-1 pathway residing upstream of TNF $\alpha$, IL-23 and IL-17.

Objectives: The goal of this study was to identify genes and pathways that regulate enthesial bone formation. SKG mice were previously reported to develop axial bone formation within 12 weeks of curdlan injection, as well as enthesial bone formation around peripheral joints ${ }^{1}$.

Methods: 9 week-old SKG or control BALB/c mice were injected IP with curdlan Histology and microCT scanning were performed at serial time points. Laser capture microscopy was performed on formalin-fixed paraffin-embedded (FFPE) tissue sections from entheseal sites around the ankle and tail vertebrae 3 weeks after curdlan injection. RNA was extracted using the Qiagen RNeasy FFPE kit and whole transcriptome analysis was performed using Affymetrix gene array MTA 1.0.

Results: Inflammation at axial entheses developed earliest at the tail base. Contrary to previous reports, we did not observe axial bone formation, but rather saw significant erosion of vertebral bodies, throughout the spine. The absence of axial bone formation was noted as late as 24 weeks post curdlan. In contrast, exuberant enthesial bone formation occurred around ankles at predictable sites of tendon/ ligament insertions into bone. We identified distinct expression of genes involved in bone resorption and formation at ankle when compared to spine enthesial sites. IPA upstream regulator analysis identified significant gene expression of upstream regulators of TGF-beta, known to recruit early osteoblast precursors to bone (z-value 2.83), and WNT3A, a WNT signalling pathway agonist that promotes osteoblast differentiation and function (z-value 2.54), in the ankle, but not the spine. These findings support the histologic observation of bone formation at ankle, but not spine, enthesial sites. In addition, significant increased gene expression of upstream regulators of the aryl hydrocarbon receptor (AhR) (z-value 2.14) was found at spine, but not ankle, enthesial sites ( $z$-value -1.78). AhR is a ligand-activated transcription factor that is a positive regulator of osteoclastogenesis via c-fos-mediated RANKL signalling.

Conclusions: Whole transcriptome analysis revealed increased expression of upstream regulators of genes in anabolic pathways, including WNT3A and TGFbeta, at ankle enthesial sites where bone formation occurs, but not at spine enthesial sites where bone erosions persist without bone formation. These pathways may thus contribute to peripheral enthesial bone formation. Significantly increased gene expression of upstream regulators of AhR, a positive regulator of osteoclastogenesis, was found at spine enthesial sites. AhR is thus a candidate regulator of continued bone resorption at spine enthesial sites in this murine model of SpA.

\section{REFERENCE:}

[1] Ruutu M, et al. $\beta$-Glucan Triggers Spondylarthritis and Crohn's Diseaselike Ileitis in SKG Mice. Arthritis Rheum 2012 Jul;64(7):2211.

Acknowledgements: This work was supported by a grant provided by AbbVie. Disclosure of Interest: None declared

DOI: 10.1136/annrheumdis-2018-eular.2973

\section{FRI0156 \\ AN ANTIMICROBIAL MECHANISM, NETOSIS, IS POTENTIALLY INVOLVED IN ANKYLOSING} SPONDYLITIS PATHOGENESIS

P. Ruiz-Limon ${ }^{1}$, M.C. Castro ${ }^{1}$, C. Lopez-Pedrera ${ }^{1}$, M.C. Abalos-Aguilera ${ }^{1}$, N. Barbarroja ${ }^{1}$, C. Perez-Sachez ${ }^{1}$, I. Arias-de la Rosa ${ }^{1}$, R. Ortega ${ }^{1}$, P. Font ${ }^{1}$, J. A. Gonzalez-Reyes ${ }^{2}$, J.M. Villalba ${ }^{2}$, C. Lopez-Medina ${ }^{1}$, A. Escudero ${ }^{1}$, E. Collantes ${ }^{1}$, Y. Jimenez-Gomez ${ }^{1} .{ }^{1}$ Rheumatology Service, IMIBIC/Reina Sofia University Hospital/Cordoba University, ${ }^{2}$ Dept. Cell Biology, Physiology and Immunology, Cordoba University; ceiA3, Cordoba, Spain

Background: Ankylosing spondylitis (AS) is a chronic inflammatory disease, of unknown etiology, that mainly affects the axial skeleton and the sacroiliac joints in the pelvis. Neutrophils are critical actors in innate immunity and their activation has been involved in the progression of AS symptoms. Recently, NETosis has been suggested to play a central role in several pathologic states, including rheumatology diseases. Nevertheless, this process has not been described yet in AS patients

Objectives: 1) To evaluate and characterise the presence of NETosis in AS patients. 2) To explore the relationship among NETosis markers and clinical characteristics of this disease

Methods: Thirty patients with AS and 32 healthy donors (HD) were included in the study. Disease activity was determined by BASDAl index and, CRP and ESR levels; in parallel, inflammatory markers were determined in plasma by ELISA kits. Spinal mobility of AS patients was measured by the BASMI index, and structural damage was calculated by the mSASSS index. Ex vivo, spontaneous NETosis generation in purified neutrophils from AS patients and HDs were measured by fluorescence $(n=6)$ and scanning electron $(n=3)$ microscopy after 6 hour of incubation. PMA, known to promote NETosis, was used as positive control. DNA extrusion was analysed by fluorescence microscopy and fluorimetry after SYTOX staining, whereas elastase percentage (NE) was analysed by fluorescence microscopy after staining of neutrophils with NE antibody. In vivo, mieloperoxidase (MPO) and NE protein expression were measured by flow cytometry (FACSCalibur), whereas extracellular DNA was examined in plasma using fluorimetry after SYTOX staining

Results: Compared to HDs, AS neutrophils showed spontaneous extracellula release of a meshwork of DNA nuclear and granule proteins (NETs), as demonstrated by fluorescence microscopy, fluorimetry, and scanning electron microscopy. Indeed, analysis of DNA fibres staining by SYTOX revealed that NETosis rate was above baseline levels after 6 hour of ex vivo AS neutrophil incubation as compared to those from HDs $(p<0.05)$. Furthermore, higher cell free-DNA levels were observed between AS patients and HDs at 6 hour $(p<0.05)$. Increased spontaneous NETs production in this pathology was additionally corroborated by the observation of an enhanced percentage of NE-staining cells after 6 hour of incubation $(p<0.05)$. In vivo, higher intracellular NE levels $(p=0.036)$, and plasma DNA concentrations ( $p=0.021)$ were also found in AS patients as compared to HDs. Correlation studies showed that plasma DNA levels positively correlated with inflammatory markers (i.e. CRP, ESR and TNF $\alpha$ ), and with the spinal mobility index BASMI. In addition, a positive correlation was found between intracellular $\mathrm{NE}$ levels and plasma IL-1 $\beta$ concentration

Conclusions: 1) NETosis is increased in AS patients. 2) Raised NETosis in AS is associated with several markers of inflammation and mobility. Thus, NETosis might act as a key mediator in the etiopathogenesis of AS. Funded by JA PI0314-2012, SER, ISCIII (RIER RD16/0012/0015)

Disclosure of Interest: None declared

DOI: 10.1136/annrheumdis-2018-eular.4291

\begin{tabular}{l|l}
\hline FRI0157 & ALTERED EXPRESSION OF THE SPLICEOSOME \\
COMPONENTS IN LEUKOCYTES SUBSETS FROM \\
PATIENTS WITH ANKYLOSING SPONDYLITIS: \\
ASSOCIATION TO DISEASE PATHOGENESIS AND \\
THERAPEUTIC RESPONSE
\end{tabular}

L. Ladehesa ${ }^{1}$, M.C. Castro ${ }^{1}$, C. Lopez-Pedrera ${ }^{1}$, R. Ortega ${ }^{1}$, S. Pedraza ${ }^{2}$, M. del Río $^{2}$, M.C. Abalos-Aguilera ${ }^{1}$, P. Ruiz-Limon ${ }^{1}$, C. Perez-Sanchez ${ }^{1}$, A. IbañezCosta $^{1}$, Y. Parra-Manso ${ }^{1}$, N. Barbarroja ${ }^{1}$, I. Arias-de la Rosa ${ }^{1}$, P. Font ${ }^{1}$, A. Escudero ${ }^{1}$, J.P. Castaño ${ }^{2}$, R.M. Luque ${ }^{2}$, E. Collantes ${ }^{1}$, Y. Jimenez-Gomez ${ }^{1}$. ${ }^{1}$ Rheumatology Service, IMIBIC/Reina Sofia University Hospital/Cordoba University; ${ }^{2}$ Dept. Biología Celular, Fisiología e Inmunología, IMIBIC/Universidad de Córdoba/Hospital Reina Sofía; CIBERobn y ceiA3, Cordoba, Spain

Background: Ankylosing spondylitis (AS) is a chronic inflammatory disease for which the etiopathogenesis has not yet been fully clarified. Splicing is a post- 
transcriptional process involved in the RNA maturation. Recent studies have revealed that a pathological deregulation of the spliceosome is associated to several human diseases. Yet, the spliceosome alterations and their modulation in therapeutic response have not been described in AS

Objectives: 1) To evaluate the potential deregulation of the spliceosome in AS leukocytes and their involvement in the disease pathophysiology. 2) To analyse the in vivo effects of anti-TNF drugs on the spliceosome components of AS leukocytes

Methods: Thirty two AS patients and 29 healthy donors (HDs) were included in a cross-sectional study. Eight AS patients were selected for a three-month longitudinal study of response to anti-TNF $\alpha$ therapy. Disease activity was determined by BASDAI index and, CRP and ESR levels. Physical function was measured by the BASFI index, spinal mobility by the BASMI index, and structural damage by the mSASSS. The expression of selected components of the major- $(n=12)$ and minor-spliceosome $(n=4)$, and splicing factors $(n=28)$ was evaluated in purified leukocyte by Fluidigm methodology; in parallel, inflammatory marker expression was determined by RT-PCR

Results: Compared to HDs, a significant deregulation in the expression of splicing factors and spliceosome components was found in lymphocytes, monocytes and neutrophils from AS patients, being neutrophils which displayed the highest number of altered molecules. Interestingly, a specific altered profile of spliceosome members was observed when compared lymphocytes (U1, U4, U5, SRSF6), monocytes (CELF4, ESRP2, RBM3, SRSF3, TIA1) and neutrophils (FBP11, SF3BTV1, U6, U12, PTB, RBM17, MAGOH, SRSF5, SRSF10). Correlation studies revealed that inflammatory profile, disease activity (CRP, ESR, BAS$\mathrm{DAI}$ ) and structural damage (BASMI, mSASSS) were associated to the alteration of a vast number of spliceosome components in all the leukocyte subsets evaluated. In addition, the BASFI index correlated with the expression of SKIP and U6atac in neutrophils.

Anti-TNF $\alpha$ treatment of selected AS patients reversed the altered expression of several spliceosome components and splicing factors in PBMCs (UA2F2, nSR100) and neutrophils (ESRP1, NOVA1, SND1, SRM160, SRSF1, CUGBP). Association studies demonstrated that disease remission was associated with reversing the altered expression of a high number of the spliceosome molecules

\section{REFERENCES:}

[1] AS patients display a deregulation of the spliceosome components associated to disease function, activity, inflammation and structural damage.

[2] Anti-TNF $\alpha$ therapy may reverse, at least partially, the altered expression of several spliceosome components and splicing factors. Alteration of the spliceosome may provide new biomarkers for disease and therapeutic response in AS. Funded:JA PI-0139-2017, ISCIII (RIER RD16/0012/0015)

Disclosure of Interest: None declared

DOI: 10.1136/annrheumdis-2018-eular.4569

\section{FRI0158 PROSTAGLANDIN E2 AND ITS RECEPTOR SUBTYPE EP4 ARE INVOLVED IN ANKYLOSING SPONDYLITIS DISEASE PROGRESSION}

A. Srinath ${ }^{1}$, N. Haroon ${ }^{1}$, G. Guggino ${ }^{2}$, I. Sari ${ }^{1}$, F. Zeng ${ }^{1}$, R. Alessandro ${ }^{2}$, A. Rizzo ${ }^{3}$ F. Ciccia ${ }^{2}$. ${ }^{1}$ University of Toronto, Toronto, Canada; ${ }^{2}$ University of Palermo;

${ }^{3}$ Azienda Ospedaliera Ospedali riuniti Villa Sofia Cervello, Palermo, Italy

Background: Single Nucleotide Polymorphisms (SNPs) in PTGER4 were found to be associated with Ankylosing spondylitis (AS) in GWAS. PTGER4 codes for the prostaglandin-E2 receptor EP4. PGE2/EP4 interaction can affect bone formation and inflammation.

Objectives: We studied serum PGE2 levels and SNPs in PTGER4 in relation to spinal fusion in AS patients. We also evaluated the interaction of smoking, PGE2 and EP4 in driving IL23 production and ILC3 functions.

Methods: Patients diagnosed with AS using the modified New York criteria and followed prospectively using a standardised protocol, were included in this study. Biological samples including serum, gut, synovial and bone marrow (BM) samples, DNA and RNA were stored and radiographs of the spine obtained every two years to assess progression. ELISA for Serum PGE2 and immunohistochemistry tissue expression of PTGS1, EP4 and pCREB were performed. Radiographs were scored by mSASSS. Patients with an increase of $\geq 1 \mathrm{mSASSS}$ unit/year on follow up were deemed progressors. Five PTGER4 SNPs, associated with AS or related diseases, call rate above $90 \%, \mathrm{MAF}>0.1$ and not in LD above 0.8 , were studied. Immune cell subsets from PBMCs were analysed for surface expression of the EP4 receptor. Additionally, PBMCs were incubated with nicotin, PGE2, or EP4 agonist to evaluate changes in ILC3 percentages and to determine cytokine expression by flow cytometry and RT-PCR.

Results: Serum PGE2 levels were significantly higher in AS progressors $(n=88)$ than in non-progressors $(n=101)(p<0.001)$. In multivariable regression analysis, there was significantly more progression in patients with higher baseline mSASSS
$(B=0.02 ; p=0.01)$ and serum PGE2 $(B=0.001 ; p=0.002)$, but lower progression with longer TNF inhibitor use $(B=-0.01 ; p=0.03)$. There was a trend towards higher progression with higher baseline ESR $(B=0.012 ; p=0.08)$. 3). A total of 172 AS patients had DNA and X-ray data for analysis. Patients with CC genotype of PTGER4 SNP rs6896969 were significantly more likely to progress compared to AA/AC (OR: $2.45,95 \% \mathrm{Cl}: 1.3$ to $4.6 ; \mathrm{p}=0.006$ ). Progressors tended to more likely be homozygous for the major allele G of the rs 4957341 SNP (OR $2.1 ; 25 \% \mathrm{Cl}$ 0.98-4.35; $\mathrm{p}=0.058$ ). Increased expression of EP4, PTGS1 and pCREB were observed in the gut, BM and synovial samples of AS patients. EP4 expression was upregulated in circulating AS monocytes and ILC3, especially smokers, and the percentage of EP4 ${ }^{+}$monocytes and ILC3 correlated with the BASDAI. Sorted $\mathrm{EP} 4^{+} \mathrm{CD} 14^{+}$cells showed a higher expression of CREB and IL-23 and in vitro stimulation of monocytes with PGE2 increased IL-23 expression, demonstrating the functional relevance of EP4 expression on monocytes. The expression of EP4 is dependent on the transcription factor AP-2a (TFAP-2a). In vitro stimulation of monocytes with nicotine induced a significant monocyte over-expression of EP4 and TFAP-2a. Finally, PGE2 stimulation of isolated PBMC from AS patients significantly expanded IL-23-producing ILC3.

Conclusions: PGE2 and its receptor EP4 are significant players in AS driving both inflammation and spinal fusion. The complex interaction of smoking, prostaglandin pathway upregulation and IL-23 dependent innate immune activation can contribute to the pathogenesis of AS

Disclosure of Interest: None declared

DOI: 10.1136/annrheumdis-2018-eular.5563

\section{FRI0159 ANTIBODIES TO POST-TRANSLATIONALLY MODIFIED COLLAGEN II IN SPONDYLOARTHRITIS}

C. Vinci ${ }^{1,2}$. ${ }^{1}$ Biochemical Pharmacology, Queen Mary University of London,

London, UK; ${ }^{2}$ Campus Biomedico of Rome, Rome, Italy

Background: Spondyloarthritis $(\mathrm{SpA})$ are a group of rheumatic diseases with either predominantly axial inflammatory symptoms of the spine and sacroiliac joints, or predominantly peripheral arthritis. The most common axial SpA (axSpA) are non-radiographic axSpA and in particular ankylosing spondylitis. The current gold standard diagnostic criteria for axSpA are clinical symptoms, radiology, MRI or ultrasound according to Assessment of SpondyloArthritis international Society (ASAS) criteria. We have previously showed that antibodies to oxidative posttranslationally modified collagen type II (oxPTM-CII) are present and specific in RA patients whether ACPA positive or negative.

Objectives: The aim of the current study was to test the presence of antibody to oxidised collagen type II (CII) in axSpA, based on the hypothesis that spina inflammation in axial $\mathrm{SpA}$ results in oxidative posttranslational modification (oxPTM) of axial joints cartilage matrix proteins such as Cll with the consequently formation of neoepitopes and a secondary humoral autoimmune response.

Methods: CIl was oxidised by exposing Cll to ribose and hypochlorous acid. Lev els of antibodies specific to native CII and CII post-translationally modified by oxidants (oxPTM-CII) was assessed by enzyme-linked immunosorbent assays (ELISA) in serum samples obtained from patients with axSpA $(n=67)$ in remission and axSpA patients $(n=14)$ non in remission. Reactivity in axSpA was compared to reactivity in samples from patients with predominantly peripheral arthritis such as psoriatic arthritis (PsA, $n=54)$ ), undifferentiated arthritis (UA, $n=49$ ) and early rheumatoid arthritis (ERA, $n=60)$. As a control we used fibromyalgia (FM, $n=19$ ) and healthy subjects $(\mathrm{HC}, \mathrm{n}=70)$. The specificity of the binding was further assessed by competitive ELISA and western blot.

Results: Stronger binding to oxPTM-Cll was observed in serum samples from axSpA patients, the positivity was $72 \%$ for patients in remission and $86 \%$ for patients not in remission (86\%) compare to positivity in PsA group (33\%), UA group $(35 \%)$ and FM group $(16 \%),(p<0.0001)$. Interestingly, binding of axSpA samples was similar to binding of serum samples from ERA (95\%). Binding to ROS-CII was directed to a range of ROS-Cll fragments between 25 and $150 \mathrm{kDa}$. Conclusions: Formation of oxPTM-Cll neoantigens in the inflamed axial joints results in an immune response that elicits antibodies specific to oxPTM-CII. Once established in future studies, antibodies to oxPTM-CII may be developed as potential biomarker for $\operatorname{axSpA}$

\section{REFERENCES:}

[1] J. Braun, J. Sieper."Ankylosing spondylitis." Lancet 2007;369(9570):1379 1390.

[2] Strollo R, Ponchel F, Malmström V, Rizzo P, Bombardieri M, Wenham CY, Landy R, Perret D, Watt F, Corrigall VM, Winyard PG, Pozzilli P, 